\title{
Ultra-Low Power Design of Wearable Cardiac Monitoring Systems
}

\author{
Rubén Braojos, Hossein Mamaghanian, \\ Alair Dias Junior*, Giovanni Ansaloni and David Atienza \\ Embedded Systems Laboratory, EPFL, Switzerland \\ Email: \{ruben.braojoslopez $\},\{$ hossein.mamaghanian $\}$, \\ \{alair.diasjunior $\},\{$ giovanni.ansaloni $\},\{$ david.atienza $\} @$ epfl.ch
}

\author{
Francisco J. Rincón, \\ Srinivasan Murali \\ SmartCardia, Switzerland \\ Email: francisco.rincon@smartcardia.com, \\ srinivasan.murali@smartcardia.com
}

\begin{abstract}
This paper presents the system-level architecture of novel ultra-low power wireless body sensor nodes (WBSNs) for real-time cardiac monitoring and analysis, and discusses the main design challenges of this new generation of medical devices. In particular, it highlights first the unsustainable energy cost incurred by the straightforward wireless streaming of raw data to external analysis servers. Then, it introduces the need for new cross-layered design methods (beyond hardware and software boundaries) to enhance the autonomy of WBSNs for ambulatory monitoring. In fact, by embedding more onboard intelligence and exploiting electrocardiogram (ECG) specific knowledge, it is possible to perform real-time compressive sensing, filtering, delineation and classification of heartbeats, while dramatically extending the battery lifetime of cardiac monitoring systems. The paper concludes by showing the results of this new approach to design ultra-low power wearable WBSNs in a real-life platform commercialized by SmartCardia. This wearable system allows a wide range of applications, including multi-lead ECG arrhythmia detection and autonomous sleep monitoring for critical scenarios, such as monitoring of the sleep state of airline pilots.
\end{abstract}

Index Terms-Wearable Embedded Systems, Bio-Medical Signal Processing, Wireless Body Sensor Nodes.

\section{INTRODUCTION AND MOTIVATION}

Embedded cardiac monitors are wearable and miniaturized devices, providing the acquisition, on-board processing and wireless transmission of cardiac bio-signals for prolonged periods of time. These wireless body sensor nodes (WBSNs) allow non-intrusive and long-term monitoring of cardiac parameters of patients, such as electrocardiogram (ECG) and

* Alair Dias Junior is also with FUMEC University, Brazil.

This work has been partially supported by the Spanish Government Research Grant TEC2012-33892, the EC FP7 FET Phidias project (Grant agreement no. 318013.), a CAPES Scholarship (grant no. 2171-13-9), and the ObeSense (no. 20NA21_143081) and BodyPoweredSenSE (no. 20NA21_143069) RTD projects evaluated by the Swiss NSF and funded by Nano-Tera.ch with Swiss Confederation financing.

Permission to make digital or hard copies of all or part of this work for personal or classroom use is granted without fee provided that copies are not made or distributed for profit or commercial advantage and that copies bear this notice and the full citation on the first page. Copyrights for components of this work owned by others than ACM must be honored. Abstracting with credit is permitted. To copy otherwise, or republish, to post on servers or to redistribute to lists, requires prior specific permission and/or a fee. Request permissions from Permissions@acm.org.

DAC '14, June 01 - 05 2014, San Francisco, CA, USA

Copyright 2014 ACM 978-1-4503-2730-5/14/06 ..\$15.00. pulse oximetry (SpO2). They represent novel solutions in the healthcare domain [1], both for the prevention of acute episodes (e.g., strokes) and for the assessment of chronic conditions (e.g., sleep disorders, stress-related pathologies).

The design of cardiac monitoring platforms is applicationdriven, because relevant data for medical examination strongly depends on the considered scenario. As an example, sleep monitoring applications involve the analysis of heart rate variability over a time window of the acquired bio-signal while the assessment of the recovery after a stroke instead requires the detailed analysis of the morphology of heartbeats. Minimizing the bandwidth on the energy-hungry wireless link [2] according to the application requirements is therefore an effective strategy for the design of ultra-low power cardiac monitors. To this end, on-node signal processing can be employed to derive the data of interest at different levels of abstraction before transmission, as illustrated in Figure 1.

This paper investigates how the energy efficiency of cardiac monitors can be enhanced by leveraging embedded processing and hardware-software optimization methodologies. We address its design space in a top-down fashion. First, cardiac applications and their requirements are introduced in Section II. Then, in Section III we detail how signal processing algorithms can support such applications and, in Section IV the challenges

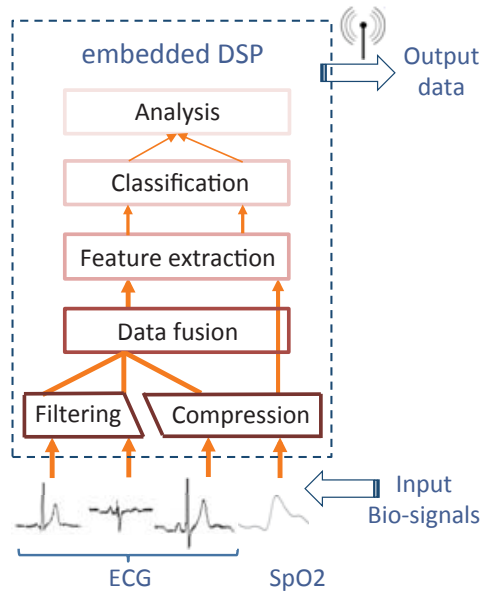

Fig. 1. On-node digital signal processing increases the energy efficiency of cardiac monitoring by rising the abstraction level and decreasing the bandwidth of transmitted data. 
involved in embedding them in resource-constrained cardiac monitors. Section V reports the performance of embedded cardiac monitors in real-world scenarios and finally section VI outlines promising future directions in this field and concludes the paper.

\section{Wireless Body SENSOR Nodes ApPliCATIONS}

While traditional cardiac monitoring equipment are bulky and target patient monitoring for short periods of time, recent advances in technology have enabled the development of ultrasmall and wearable monitoring devices. With the development of embedded cardiac monitors, we can envision a new generation of wearable systems where different sensors are integrated directly in the user's dress, processing and streaming the vital signs information to a mobile phone or a cloud server.

This opens up new application domains for cardiac monitoring, ranging from extracting behavioral information of the user (mental and emotional state), sleep/fatigue information and early detection and prevention of diseases.

The digital signal processing required for such different application scenarios varies widely. Applications that extract behavioural information typically only require processing of beat-to-beat intervals, while the diagnosis of heart problems requires the processing of more detailed morphological information of each heartbeat. Applications such as driver sleep monitoring require classifiers that are trained over different population sets and driving scenarios, and ported onto embedded micro controllers. The noise level of the signal and the required filtering algorithms also vary based on the application. For a cardiac system embedded in vehicles that use noncontact sensors, a major challenge is to remove the common mode electrical noise and obtain a clean signal, while for ambulatory monitoring of stroke patients, the challenge is to remove muscular and motion artifacts. Towards this end, in this paper, we present an application driven methodology for cardiac and bio-signal processing.

\section{CARDIAC SIGNAL PROCESSING METHODS}

As illustrated in Figure 1, digital signal processing on embedded cardiac monitors typically follows a number of phases. A filtering stage is mandatory before performing further processing steps. To further counteract noise, different signals with the same modality (e.g.: multi-lead ECGs) can be combined after filtering. Afterwards, characteristic features are derived from the the cardiac signals with processes such as ECG delineation, and classification is performed to detect abnormalities in each heart-beat. Information at each level is instrumental to perform automated diagnosis for different cardiac-related pathologies.

An alternative solution, also effective in minimizing transmission bandwidth, is to compress the signal on the cardiac monitor, and reconstruct it for further analysis on the receiver side. The two approaches are not mutually exclusive. In [3], in fact, a classification methodology on compressed ECG signals has been proposed.

This section illustrates promising solutions for the automation of the different phases.

\section{A. ECG compression}

Today's state-of-the-art WBSN-enabled ambulatory ECG monitors still fall short of the required energy efficiency and longevity. This is mainly because of the raw data transfer over energy-hungry wireless links. It is today acknowledged that the achievement of truly WBSN-enabled ambulatory monitoring systems requires more breakthroughs not only in terms of ultra-low-power read-out electronics and radios, but also in terms of dedicated digital processors, which execute the associated embedded feature extraction and data compression algorithms in order to reduce airtime over wireless links.

In [4], the potential of the compressed sensing (CS) signal acquisition/compression paradigm for low-complexity energyefficient ECG compression has been investigated. It is shown that CS could outperform its state-of-the-art counterpart compression algorithms, thanks to its low complexity and CPU execution time in terms of overall energy efficiency. With more advances on the reconstruction algorithm, in [5], the possibility of a real-time CS decoder running on an iPhone (acting as a WBSN coordinator) has been demonstrated.

Building on these initial works which proves the suitability of using CS for ECG compression on the resource- and energy-aware WBSN, the technique has been extended to fully leverage and exploit underlying structural information, like any state-of-the-art compression technique. More specific recovery algorithms for single lead and multi-lead ECG has been presented in [6].

Beyond all these works, CS is usually used as a very low cost and easy to implement compression technique. Signals should be acquired with the traditional limitations on the bandwidth (BW) and after the major portion of redundant data should be discarded. The main challenges are then taking the whole design of the front-end and read-out devices to the next level based on the promises of the CS and merging the sampling and compression steps. This removes a large part of the digital architecture and considerably simplifies analog-toinformation (A2I) conversion devices.

This so-called "analog CS", where compression occurs directly in the analog sensor readout electronics prior to analogto-digital conversion, could thus be of great importance for applications where bandwidth is moderate, but computationally complex, and power resources are severely constrained. Different realization of the CS-based "analog-to-information" readout devices has been introduced in the literature [7], [8], although designing a truly CS-based A2I still remains as a challenge.

\section{B. Noise filtering and source combination}

Cardiac bio-signals are usually affected by noise, which must be filtered before relevant features can be retrieved from the acquisitions. Different sources of noise range from those coming from environmental factors (e.g. electromagnetic interference) to others of biological nature (e.g. muscular activity). The authors of [9] propose a filtering technique based on the application of two morphological operators (erosion and dilation), which removes unwanted components from 


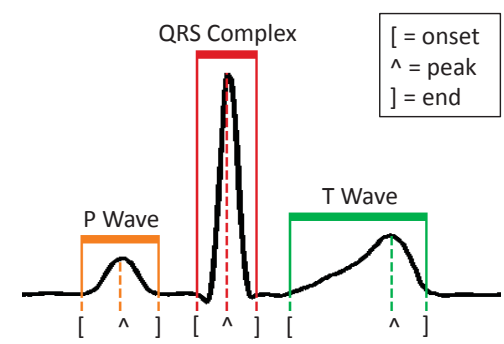

Fig. 2. Delineated normal sinus beat.

the input signal. Alternatively, [10] proposes a technique for removing low-frequency components causing the so called baseline wandering. This method, based on cubic splines, searches for "knots" in a characteristic silent region of the acquired signal (before each QRS complex), and interpolates three consecutive knots to estimate the baseline.

In [11], it is shown that the effect of noise can be also reduced by combining different ECG leads before the analysis and/or delineation phase. Simple root mean square (RMS) aggregation of inputs, is presented as a light-weight, yet effective, implementation strategy.

\section{ECG Delineation}

ECG delineation is the process of identifying the fiducial points (start, peak and end) of the characteristic waves composing each heart-beat (Figure 2). The information enables the diagnosis of a large set of cardiac conditions, such as arrhythmias. For this purpose, [12] propose a method based on wavelet decomposition, relying on the fact that different waves present distinct frequency components.

An alternative strategy, presented in [13], proposes to use a morphological transform of the ECG to automate the delineation of fiducial points. The method gives an effective solution to the problem of delineation, as minima in the transformed signal indicates the presence of peaks in the original wave, while maxima (or sudden changes in slope) delimit the start and end point of each wave.

\section{Embedded classification}

Higher-level ECG processing, after the delineation phase, can further reduce the amount of data to be transmitted, while also adding further information about the processed signal. The work presented in [14] proposes the utilization of a classifier to identify abnormal heartbeats and trigger delineation only in those cases. The authors describe a methodology to implement a fuzzy network into a state-of-the-art WBSN meeting realtime and sensitivity constraints.

An important problem in classification is to identify a set of representative features for each heartbeat. The random projection approach introduced in [15] is particularly promising in this context. It allows the minimization of the number of features (and therefore a simplified classification process) as well as the efficient computation of the feature set.

\section{EMBEDDED CARDIAC MONITORING PLATFORMS}

The design of a truly effective embedded cardiac monitor has to overcome diverse challenges. The most important design goals are to require low maintenance and allow long-term execution, at the same time causing as little discomfort as possible to subjects. Moreover, accurate diagnostic data should be retrieved for medical evaluation.

These conflicting goals require a careful system-wide evaluations in the platform design. Energy efficiency is a key objective, as it allows to minimize the battery size and weight while retaining continuous operations for extended periods of time. This section describe several effective strategies (both at the hardware and software levels) allowing ultra-low power regimes while performing embedded processing.

Moreover, physical constrains impede the continuos acquisition of important cardiac parameters. We show how parameters such as Blood Pressure (BP) can instead be estimated from other ready-obtainable quantities.

\section{A. Software optimizations}

The filtering, delineation and classification algorithms proposed in Section III must be carefully tailored to be executed on resource-constrained embedded cardiac monitors. Typically, these platforms (like the SmartCardia device presented in Figure 4) operate at a clock frequency of few $\mathrm{MHz}$ and only support integer arithmetic operations.

Computationally-intensive parts of applications must therefore be analyzed to derive light-weight implementations. This approach is exemplified in [12], where a proper choice of the filter bank coefficients leads to an efficient execution of the wavelet-based delineation on a state-of-the-art embedded device. In the cases of morphological filtering and delineation, if a flat structuring element is employed, the computational demands of the morphological operations can be drastically reduced by keeping track of only the center value, maximum and minimum in a sliding window of the input signal.

The approximation of complex functions is also as an effective strategy to reduce algorithmic complexity. For instance, in the scenario of heartbeat classification, which usually involves the evaluation of many gaussian functions, a foursegments linearization is shown to achieve close-to-optimal results [14], while vastly simplifying the computational requirements. Moreover, by using random projections (described in Section III-D) memory usage can be minimized considering a projection matrix only composed by elements of value 0 , 1 and -1 , which can be represented using only two bits per component.

Sparsity considerations are important to minimize computation and memory usage. In [16], it is shown how few nonzero elements in the sensing matrix suffice to achieve close-tooptimal results when performing compressive sensing, while minimizing the run-time workload.

Along the same lines, the knowledge of the structure of coefficient vectors can be exploited to increase the quality of compressed sensing by differentiating signal information from recovery artifacts. A first approach stems from the observation that wavelet coefficients are naturally organized into a tree structure, and the largest coefficients cluster along the branches of this tree. A CS reconstruction algorithm based on the 


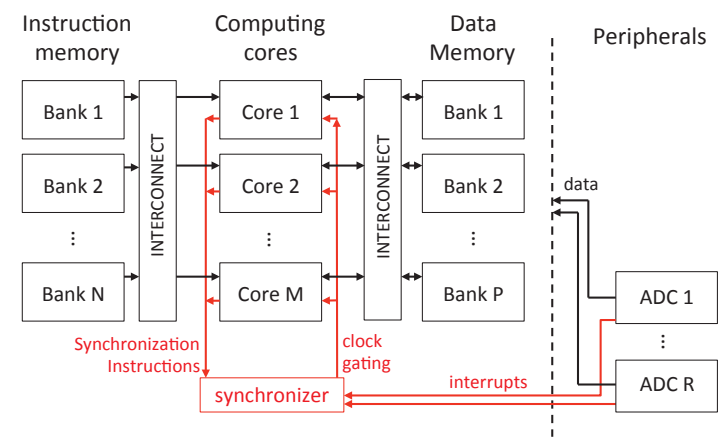

Fig. 3. Hardware architecture of a typical multi-core WBSN. In red, hardware support for synchronization.

connected tree model has been proposed in [17]. Second, in case of the multi-lead ECG compression, there is a strong correlation between the sparsity structure among the leads, each lead therefore conveying useful information about other leads. In particular, non-zero coefficients are partitioned in subsets or groups, and this information can be employed to enhance the compression performance across all leads [6].

\section{B. Ultra-low-power architectures for cardiac monitoring}

Cardiac monitoring applications present a high degree of parallelism, either because multiple sources are processed by the same algorithms (e.g., filtering of multiple ECG inputs) or because processing is divided into consecutive different phases of computation (as introduced in Figure 1). These characteristics allow the parallelization of the workload on a multi-core core architecture by exploiting voltage scaling to achieve substantial energy savings.

In [18] we propose a platform interfacing multiple processors to independent multi-bank program and data memories (cf. Figure 3). Low-overhead mechanisms are introduced to synchronize code execution and enable single-instruction multiple-data (SIMD) operations, resulting in a decrease of the energy consumption of the instruction memory subsystem. To this end, the employed broadcasting mechanism, which is implemented by the interconnect networks, merges multiple identical read requests from different cores into a single memory access. Moreover, this architecture includes a software technique based in barrier insertion to maintain cores in lock-step and recover from de-synchronization after data dependent branches. This technique requires a reduced instruction set extension that annotates the synchronization status on dedicated data words stored in the shared memory to allow the synchronization hardware to properly orchestrate the execution flow. This architecture enables the use of producerconsumer relationships among computational stages and the application of the proposed methodology allows to correctly map bio-medical applications onto the multi-core platform in order to avoid program memory conflicts, and therefore unnecessary stalls and performance degradation. Interestingly, fine-tuned load balancing is not a necessary precondition for energy efficiency in cardiac monitoring systems.

A complementary approach for minimizing power consumption in this kind of devices is to include application-specific accelerators. In the case of compressed sensing, the authors of [19] highlight that a minimal hardware support accompanied by a specific instruction set extension of a RISC core can achieve more than ten-fold power saving with respect to a baseline implementation while performing compressed sensing over an ECG signal.

\section{Real-time estimation of multiple cardiac parameters}

In clinical scenarios, information extracted from the ECG is analyzed along with other cardiac bio-signals, such as blood pressure (BP) and pulse oximetry, to assess the global health status of the cardiovascular system. The realization of multi-modal cardiac monitors integrating diverse sensors in a wearable device is, therefore, desirable but not straightforward. As an example, sensors capable of directly measuring blood pressure are either cumbersome (e.g., inflating cuffs) or extremely complex (e.g., arterial tonometry).

An interesting approach for multi-modal analysis is to combine available information to estimate parameters which cannot be easily measured. For instance, the pulse arrival time (PAT), calculated using ECG and a simple and inexpensive photoplethysmograph (PPG) finger probe, can be used to estimate the pulse wave velocity (PWV), which is a surrogate marker for arterial stiffness and BP [20].

In addition, when signals of multiple modalities are considered, the correlation between the different inputs can be used to facilitate signal processing. Most cardiac bio-signals originate from the response to the bioelectric stimuli reflected in the ECG. The signals are, therefore, time-locked to these stimuli. This information can be used to remove noise (which is instead uncorrelated to the stimuli) with different techniques such as ensemble averaging (EA) and adaptive impulse correlated filtering (AICF). In [21] it is shown that ECG information can be employed to calculate, among other parameters, the EA of the pulse oximetry. Also, AICF can be used to filter the ECG signal [22] and to de-noise PPG signals [23]. The disadvantage of using EA is that the beat-to-beat variation of the signals is lost after the processing. AICF, on the other hand, is also capable of tracking dynamic changes in the signal.

\section{EXPERIMENTAL RESULTS}

Embedded cardiac monitors enable a new family of application scenarios in the healthcare domain. Although they are computationally- and energy- constrained, these devices are able to perform the advanced signal processing algorithms described in Sections III and IV, allowing novel breakthroughs in the healthcare monitoring field. As an example, the SmartCardia device [24] depicted in Figure 4 is able to perform 3-lead ECG monitoring and execute a set of algorithms for real-time ECG filtering and arrhythmia detection. In particular, it allows the automatic detection of abnormal cardiac events and the remote notification of such phenomena to a centralized server infrastructure, thanks to an on-board radio transceiver. Compressed Sensing is employed to efficiently transmit excerpts of the acquired signals, periodically or when an abnormality is detected. This SmartCardia device embeds 


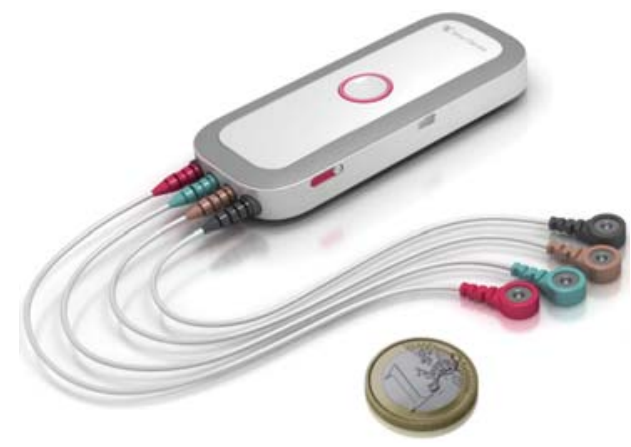

Fig. 4. SmartCardia 3-lead ECG monitoring device.

an ultra-low-power micro-controller for digital processing, an acquisition front-end and a wireless transmission stage, all in a compact form factor. The mean time between charges is typically one week.

This level of functionality is achieved by an optimized implementation of hardware and software components, such as the ones described in this paper. Moreover, these optimizations do not have major impact on the obtained quality of the retrieved data. As an example, the performance of the illustrated ECG delineation algorithms are in line with the results reported by computing-demanding off-line variants, while requiring only a fraction of the resources $(7 \%$ of the duty cycle and $7.2 \mathrm{kB}$ of memory [12]). For this application, the measured sensitivity and specificity of retrieved fiducial points are above $90 \%$ in all cases, which is at the target level for medical use in this field.

Figure 5 compares the averaged signal-to-noise ratio (SNR) results over different compression ratios $(C R)$ for singlelead and multi-lead CS compression [6]. These results show that an averaged SNR over $20 d B$ (corresponding to good reconstruction quality [16]) is reached for $C R=65.9 \%$ and $C R=72.7 \%$ for single and multi-lead CS, respectively. To characterize the power figure, the compression algorithms are implemented on our target WBSN running FreeRTOS on a 16-bit processor and simple medium access control (MAC) scheme for wireless communication (IEEE 802.15.4) between the node and the base station. Figure 6 depicts the share of radio, OS and compression in the total node power consump-

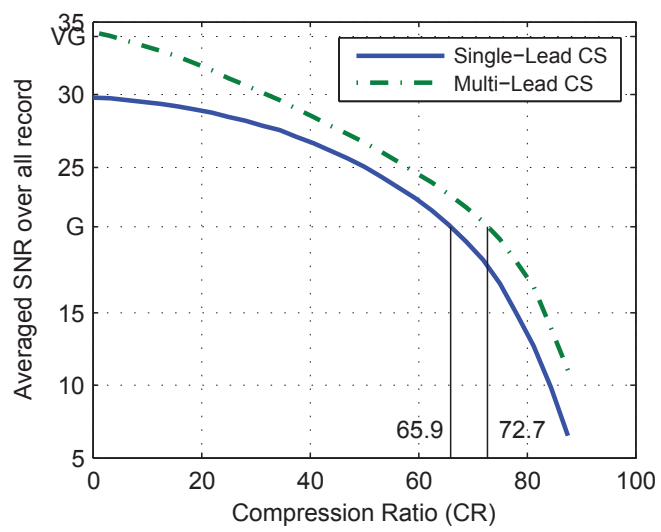

Fig. 5. Output averaged SNR over all records over different compression ratios for single-lead and multi-lead compression.

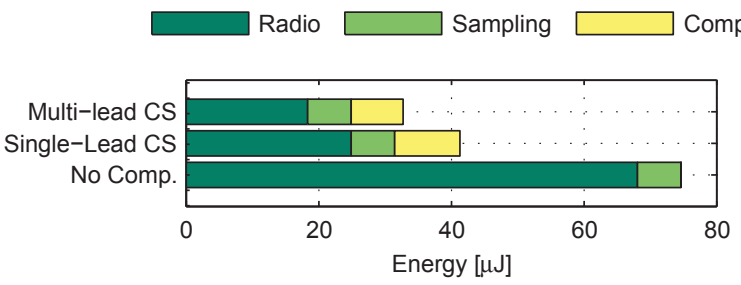

Fig. 6. Breakdown of energy consumption for our target platform.

tion for raw data streaming and two CS-based compression for good quality of reconstruction. The average power reduction estimates are $44.7 \%$ and $56.1 \%$ compared to raw-data streaming for single-lead and multi-lead CS compression. This proves the suitability of CS as a promising low-power compression technique for wearable cardiac monitoring systems.

In addition, similar results have been obtained for applications performing a diagnosis at a higher level of abstraction, such as Atrial Fibrillation (AF) detection [25]. This cardiac monitoring application uses the results of the ECG delineation to analyze the regularity of the heart beat rate as well as the shape of the $\mathrm{P}$ wave, which constitute two characteristic irregularities of $\mathrm{AF}$ episodes. The results generated after the observation of these irregularities can be subsequently analyzed in real-time using a fuzzy classifier. This low-complexity approach achieves $96 \%$ sensitivity and $93 \%$ specificity, which are comparable figures to state-of-the-art off-line AF detection algorithms while operating in real-time on an embedded device.

Finally, the use of multi-core computing architectures in cardiac monitoring systems (cf. Section IV-B) increases further the energy efficiency of the processing stage. In particular, Figure 7 shows how the filtering (3L-MF), delineation (3LMMD) and classification (RP-CLASS) applications described in Section III can be efficiently executed on the presented multi-core platform of Figure 3 (MC), reducing up to $40 \%$ the global power consumption with respect to a single-core variant (SC).

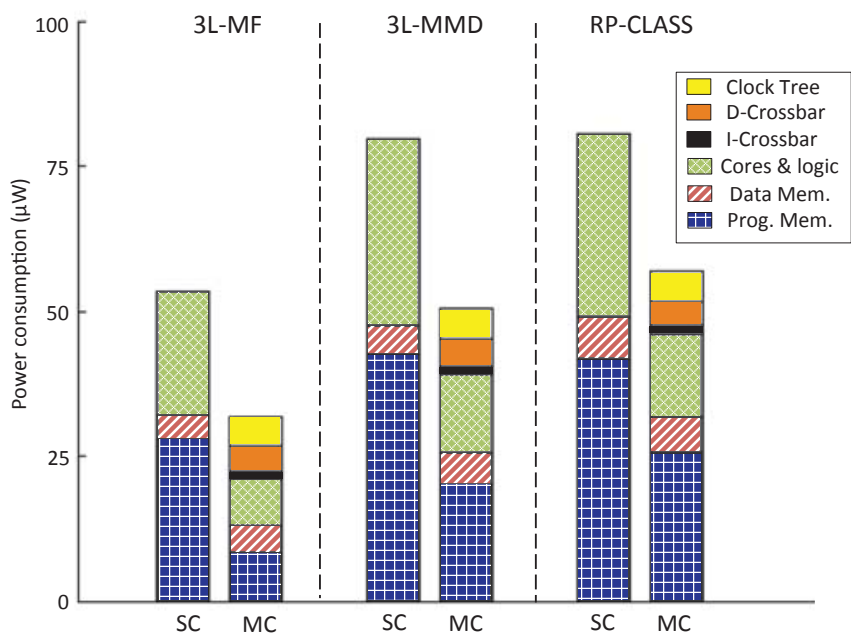

Fig. 7. Average power consumption decomposition of a synchronized multicore (MC) system and an equivalent single-core (SC) architecture. 


\section{Conclusions And Future Applications}

In this paper we have presented the main design challenges and latest approaches targetting next-generation ultralow power wearable cardiac monitors, which need to perform an energy-efficient real-time acquisition and processing of ECG, thus providing the possibility of performing on-line diagnosis and analysis of the cardivascular state of a person. In order to develop this new cardiac monitoring systems, we have shown that multiple optimizations exploiting the target bio-signal features need to be performed at both hardware and software levels in order to achieve the target ultra-low power figures needed to extend autonomy of the systems for longterm bio-signals monitoring.

As a result, these new ultra-low power cardiac monitoring WBSNs will be able to enable novel medical approaches for the diagnosis of diverse ailments. In particular, a promising direction to be investigated in the near future is related to neurodegenerative disorders, such as, Alzheimer Desase (AD). Even though the mechanisms linking the autonomic regulation of the heart activity to $\mathrm{AD}$ are not fully understood, recent studies have demonstrated a correlation between neurological degeneration and aberrant ECG signals, indicating the latter as a potentially useful bio-marker of AD progression [26].

Moreover, researchers have explored the neural basis of bodily self-consciousness and have showni in very recent studies that a tight relationship exists between the brain processes and the body states, such as, the heartbeat [27]. Therefore, expected development of wearable cardiac monitors has the potential to open up several new applications in neurorehabilitation by exploiting the relationship between the brain processes and cardiac factors, as well as enabling effective mind training and improvement of children with learning disabilities.

\section{REFERENCES}

[1] Y. Hao and R. Foster, "Wireless body sensor networks for healthmonitoring applications," Physiological Measurement, vol. 29, no. 11, p. R27, Oct. 2008.

[2] F. Chen, F. Lim, O. Abari, A. Chandrakasan, and V. Stojanovic, "Energyaware design of compressed sensing systems for wireless sensors under performance and reliability constraints," Circuits and Systems I: Regular Papers, IEEE Transactions on, vol. 60, no. 3, pp. 650-661, Mar. 2013.

[3] M. Shoaib, K. Lee, N. Jha, and N. Verma, "A 0.6 -107 uw energyscalable processor for directly analyzing compressively-sensed eeg," Circuits and Systems I: Regular Papers, IEEE Transactions on, vol. PP, no. 99, pp. 1-14, 2014.

[4] H. Mamaghanian, N. Khaled, D. Atienza, and P. Vandergheynst, "Compressed sensing for real-time energy-efficient ecg compression on wireless body sensor nodes," Biomedical Engineering, IEEE Transactions on, vol. 58, no. 9, pp. 2456 -2466, sept. 2011.

[5] K. Kanoun, H. Mamaghanian, N. Khaled, and D. Atienza, "A realtime compressed sensing-based personal electrocardiogram monitoring system," in Conference on Design, Automation and Test in Europe (DATE' 11), Sep. 2010.

[6] D. A. Hossein Mamaghanian, Giovanni Ansaloni and P. Vandergheynst, "Power-efficient joint compressed sensing of multi-lead ecg signals," in IEEE International Conference on Acoustics, Speech, and Signal Processing (ICASSP), 2014.

[7] Y. Eldar, "Analog compressed sensing," in Acoustics, Speech and Signal Processing, 2009. ICASSP 2009. IEEE International Conference on, April 2009, pp. 2949-2952.
[8] H. Mamaghanian, N. Khaled, D. Atienza, and P. Vandergheynst, "Design and exploration of low-power analog to information conversion based on compressed sensing," Emerging and Selected Topics in Circuits and Systems, IEEE Journal on, vol. 2, no. 3, pp. 493-501, 2012.

[9] Y. Sun, K. L. Chan, and S. M. Krishnan, "ECG signal conditioning by morphological filtering," Computers in Biology and Medicine, vol. 32 no. 6, pp. 465-479, Sept. 2002.

[10] C. Meyer and H. Keiser, "Electrocardiogram baseline noise estimation and removal using cubic splines and state-space computation techniques," Computers and Biomedical Research, vol. 10, no. 5, pp. 459470, Oct. 1977.

[11] R. Braojos, G. Ansaloni, D. Atienza, and F. Rincon, "Embedded realtime ecg delineation methods: A comparative evaluation," in Bioinformatics Bioengineering (BIBE), 2012 IEEE 12th International Conference on, Nov 2012, pp. 99-104.

[12] F. Rincon, N. Boichat, D. Atienza, and N. Khaled, "Wavelet-based ECG delineation on a wearable embedded sensor platform," in Wearable and Implantable Body Sensor Networks, 2009. BSN 2009. Sixth International Workshop on, June 2009, pp. 256-261.

[13] Y. Sun, K. Luk Chan, and S. Muthu Krishnan, "Characteristic wave detection in ECG signal using morphological transform," BMC Cardiovascular Disorders, vol. 5, pp. 1-7, Sep 2005.

[14] R. Braojos, G. Ansaloni, and D. Atienza, "A methodology for embedded classification of heartbeats using random projections," in Design, Automation Test in Europe Conference Exhibition (DATE), 2013, March 2013, pp. 899-904.

[15] D. Achlioptas, "Database-friendly random projections: JohnsonLindenstrauss with binary coins," Journal of Computer and System Sciences, vol. 66, no. 4, pp. 671- 687, June 2003.

[16] H. Mamaghanian, N. Khaled, D. Atienza, and P. Vandergheynst, "Compressed sensing for real-time energy-efficient ecg compression on wireless body sensor nodes," Biomedical Engineering, IEEE Transactions on, vol. 58, no. 9, pp. 2456-2466, Sept 2011.

[17] M. F. Duarte, M. B. Wakin, and R. G. Baraniuk, "Fast reconstruction of piecewise smooth signals from incoherent projections," in Signal Processing with Adaptative Sparse Structured Representations (SPARS' 05), November 2005.

[18] R. Braojos Lopez, I. Beretta, G. Ansaloni, and D. Atienza Alonso, "Hardware/Software Approach for Code Synchronization in Low-Power Multi-Core Sensor Nodes," in Proceedings of the IEEE/ACM 2014 Design Automation and Test in Europe (DATE) Conference, vol. 1, no. 1. IEEE/ACM Press, 2014, pp. 50-55.

[19] J. Constantin, A. Dogan, O. Andersson, P. Meinerzhagen, J. Rodrigues, D. Atienza, and A. Burg, "Tamarisc-cs: An ultra-low-power applicationspecific processor for compressed sensing," in VLSI and System-onChip (VLSI-SoC), 2012 IEEE/IFIP 20th International Conference on, Oct 2012, pp. 159-164.

[20] H. Gesche, D. Grosskurth, G. Kuchler, and A. Patzak, "Continuous blood pressure measurement by using the pulse transit time: comparison to a cuff-based method," European Journal of Applied Physiology, vol. 112, no. 1, pp. 309-315, Jan 2012.

[21] J. M. Solaï,$\frac{1}{2}$ i Caros, "Continuous non-invasive blood pressure estimation,” Ph.D. dissertation, ETH Zurich, 2011.

[22] P. Laguna, R. Jane, O. Meste, P. Poon, P. Caminal, H. Rix, and N. Thakor, "Adaptive filter for event-related bioelectric signals using an impulse correlated reference input: comparison with signal averaging techniques," Biomedical Engineering, IEEE Transactions on, vol. 39, no. 10 , pp. 1032-1044, Oct 1992.

[23] K. Pilt, K. Meigas, J. Lass, M. Rosmann, and J. Kaik, "Adaptive impulse correlated filter (aicf) improvement for photoplethysmographic signals," in Engineering in Medicine and Biology Society, 2008. EMBS 2008. 30th Annual International Conference of the IEEE, Aug 2008, pp. 273-276.

[24] SmartCardia. [Online]. Available: http://smartcardia.com/

[25] F. Rincon, P. Grassi, N. Khaled, D. Atienza, and D. Sciuto, "Automated Real-Time Atrial Fibrillation Detection on a Wearable Wireless Sensor Platform," in Proceedings of 34th IEEE Annual International Conference of the Engineering in Medicine and Biology Society (EMBC 2012), 2012, pp. 2472-2475.

[26] M. Vilhena Toledo and J. Junqueira, LuizFernando, "Cardiac autonomic modulation and cognitive status in alzheimer's disease," Clinical Autonomic Research, vol. 20, no. 1, pp. 11-17, Feb. 2010.

[27] O. Blanke, "Multisensory brain mechanisms of bodily selfconsciousness," Nature Reviews Neuroscience, vol. 13, no. 8, pp. 556-571, 2012 